\title{
Wall shear stress from a single almost spherical and a Taylor bubbles in laminar upward tube flow
}

\author{
Leonid Timkin ${ }^{*}$ and Roman Gorelik \\ Kutateladze Institute of Thermophysics, Pr. Ac. Lavrentev, 1, 630090 Novosibirsk, Russia
}

\begin{abstract}
An experimental electrodiffusional technique with eight double probes is used to detect perturbation the wall shear stress caused by a single bubble in laminar upward tube flow. A small almost spherical and different length Taylor bubbles are considered. The shear stress perturbations by bubbles have a complex structure. It is possible to define three components of perturbation caused by a small bubble. The perturbation by Taylor bubble contains only two components due to the main flow symmetry around the bubble. An unexpectedly long shear stress pulsations zone is registered behind the bubbles.
\end{abstract}

\section{Introduction}

Anomalously high wall shear stress in bubbly flow may increase the heat-mass transfer and result in the fast degradation of an industrial apparatus. Numerous works have been devoted to this phenomenon. Many investigations have been dedicated to rising Taylor bubbles, starting from $[1,2]$. One of the last review of slug flows focused on the formalization of flow governing laws and the physical mechanisms involved, was given in [3]. In the last few decades, new techniques have been used to deeper understanding of bubble rising. The growing capabilities of data acquisition and processing, along with higher precision of measurement techniques, have resulted in a new understanding of flow features. The rising of single bubble even in laminar upward flow is complex and asymmetric, sometimes including liquid flow reversals. The rising bubbles induce different component of flow perturbations, that may prevail under the various flow conditions, changing the integral flow characteristics. The estimation of different components of bubble induced pertuberation is essential for real flow modeling. This work aims at estimating the single bubble impact on the wall shear stress in laminar flow.

\section{Experimental setup}

The experimental setup consist of a vertical tube with inner diameter $D=14.8 \mathrm{~mm}-$ inner diameter, and $6.0 \mathrm{~m}$ in height, with a top and a bottom tank, a pump, a downward tube, and system of thermostabilization. A bubble is generated at the tube inlet in special tank filled

* Corresponding author: timkin@itp.nsc.ru 
with liquid. A simple rotated cap is filled with air and overturned to produce a single bubble. Then, the bubble is carried by the flow into the tube. The test section was $5 \mathrm{~m}$ distant from the tube inlet. An immersion block with optical windows and a glass tube inside were installed at the top of the test section to take pictures of moving bubbles. A rotameters were used for liquid flow control. A ferriferrocyanide electrochemical solution with glycerin addition to increase the viscosity, was used as the test liquid. At a temperature of $20 \pm$ $0.2^{\circ} \mathrm{C}$ the liquid density was $\rho=1125 \mathrm{~kg} / \mathrm{m}^{3}$, the kinematic viscosity $v=3.5 \cdot 10^{-6} \mathrm{~m}^{2} / \mathrm{s}$.

Wall shear stress measurements were performed using an electrodiffusional technique. The eigth double electrodiffusional probes glued into the wall of the test section uniformly over the tube perimeter. Each probe consists of two rectangular platinum electrodes with a thin insulation layer $(20 \div 30 \mu \mathrm{m})$ in between. In experiments with Taylor bubbles, the size of the electrodes was $35 \times 210 \mu \mathrm{m}$, for small bubbles the size of the electrodes was $40 \times 700 \mu \mathrm{m}$. The probes were connected to a computer through individual amplifiers and ADC. The detail of

(a)

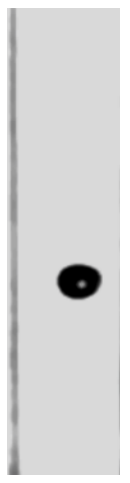

Fig. 1. Photos: spherical bubble, $R e=208$ $R e_{\mathrm{b}}=264, d_{\mathrm{b}}=4.95 \mathrm{~mm}, V_{\mathrm{b}}=$ $0.24 \mathrm{~m} / \mathrm{s}$, (b) short Taylor bubble, $R e=954, L_{\mathrm{b}}=0.041$ $\mathrm{m}, V_{\mathrm{b}}=0.462 \mathrm{~m} / \mathrm{s}$, (c) long Taylor bubble, $R e=1387, L_{\mathrm{b}}$ $=0.51 \mathrm{~m}, V_{\mathrm{b}}=0.67 \mathrm{~m} / \mathrm{s}$.

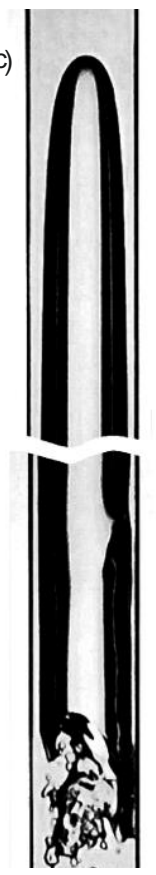

probes calibration is fixed in [4]. Photos of bubble types checked in experiments are presented on Fig. 1. The flow characterized by: a superficial liquid velocity $V_{1}$, a liquid Reynolds number $R e=D \cdot V_{1} / v$, a bubble's rising velocity $V_{\mathrm{b}}$, a small bubble diameter $d_{\mathrm{b}}$, terminal velocity $V_{\infty}$, Reynolds number $R e_{\mathrm{b}}=d_{\mathrm{b}} \cdot V_{\infty} / v$, and a Taylor bubble length $L_{\mathrm{b}}$.

\section{Results}

The shear stress perturbations by small bubbles are presented in Fig. $2(\mathrm{a}-\mathrm{c})$. Prior to the bubble appearance, the shear stress resembles a smooth surface without pulsations, with the shear stress corresponding to single-phase flow. The bubble induces a perturbation of this surface. It is possible to define three components of the perturbation. The first component is a decrease in shear stress, which is caused by an accelerated liquid around the bubble in the direction opposite to the bubble motion. The second component is an intense positive peak. The small bubbles rise out of the tube axis, move closer to one side of the tube wall. In the gap between the bubble and tube wall, the liquid is carried by bubble with high velocity, and generating a peak structure. This peak was previously detected in $[5,6]$. The similar structure was obtained in $[7,8]$. The third component is a positive pulsations behind the bubble. This component presents a gradual decay of the perturbed shear stress to a singlephase laminar value. In deep laminar flow this component is small, Fig. 2 (a). For higher Reynolds numbers $R e=988$ and 1920 the peak component grows and disintegrates into a several peaks, which are distributed over the whole tube perimeter. The peak and pulsation component join together to form a turbulent pulsations zone, completely occupying the tube crossection. At higher liquid Re numbers they are responsible for the anomalously high shear stress in two-phase bubbly flows. There are tested long Taylor bubbles $\left(L_{\mathrm{b}} \sim 0.25 \div\right.$ $0.65 \mathrm{~m})$ and short ones $\left(L_{\mathrm{b}} \sim 0.04 \div 0.2 \mathrm{~m}\right)$. Three wall shear stress perturbations caused by Taylor bubbles of length $L_{\mathrm{b}} \sim 0.5 \mathrm{~m}$ are presented in Fig. $2(\mathrm{~d}-\mathrm{f})$. 


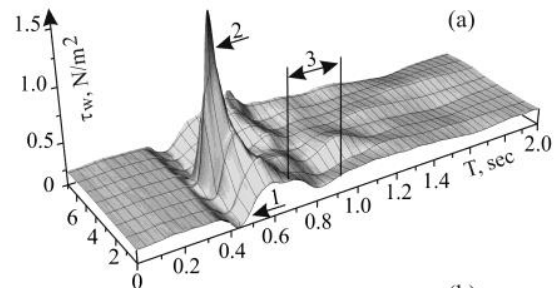

(b)

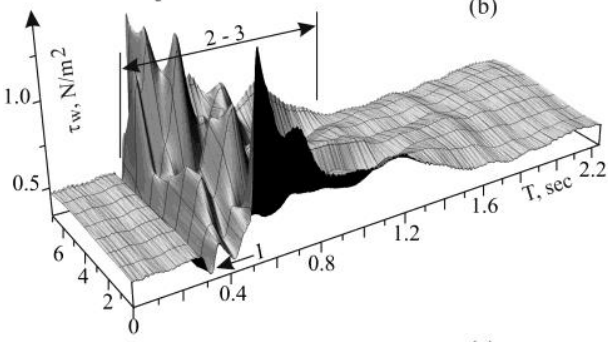

(c)

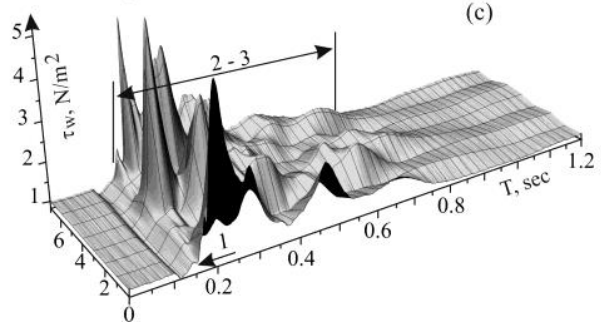

(d)

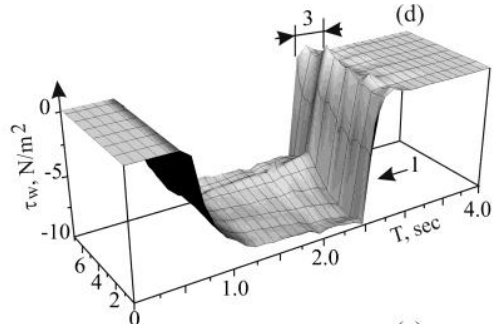

(e)

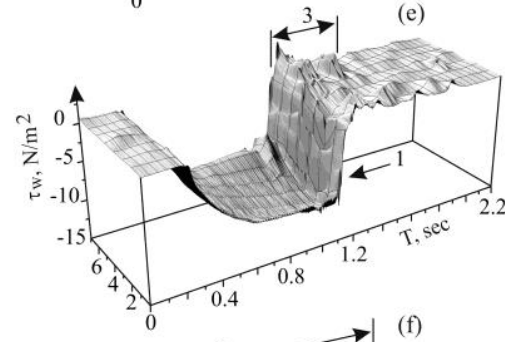

(f)

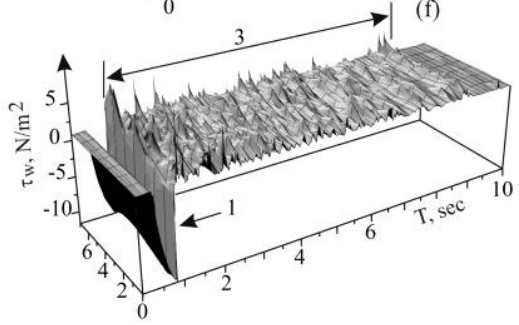

Fig. 2. The perturbations caused by almost similar small bubbles: (a) $R e=444, R e_{\mathrm{b}}=272, d_{\mathrm{b}}=5.15$ $\mathrm{mm}, V_{\mathrm{b}}=0.33 \mathrm{~m} / \mathrm{s} ;$ (b) $R e=988, R e_{\mathrm{b}}=250, d_{\mathrm{b}}=4.63 \mathrm{~mm}, V_{\mathrm{b}}=0.503 \mathrm{~m} / \mathrm{s} ;$ (c) $R e=1920, R e_{\mathrm{b}}=280$, $d_{\mathrm{b}}=5.36 \mathrm{~mm}, V_{\mathrm{b}}=0.865 \mathrm{~m} / \mathrm{s}$. The perturbations caused by almost similar Taylor bubbles: (d) $R e=$ $217, L_{\mathrm{b}}=0.48 \mathrm{~m}, V_{\mathrm{b}}=0.204 \mathrm{~m} / \mathrm{s}$; (e) $R e=954, L_{\mathrm{b}}=0.46 \mathrm{~m}, V_{\mathrm{b}}=0.48 \mathrm{~m} / \mathrm{s}$; (f) $R e=1844, L_{\mathrm{b}}=0.48$ $\mathrm{m}, V_{\mathrm{b}}=0.87 \mathrm{~m} / \mathrm{s}$. Where: 1 - decreasing component, 2 - peak component, $2-3$ and 3 -pulsations zone.

Taylor bubble perturbations contain only two components which are the decreasing and the pulsations ones. The peak component is absent due to the main flow symmetry. Again, in front of bubble the shear stress is constant over the tube perimeter. The Taylor bubble pushes the shear stress into the negative values. A downwardly accelerated liquid film in a ring gap between the bubble and the tube wall forms the strong decreasing component. The readings of all probes are almost the same. This proves the fact that the flow around the Taylor bubble is symmetrical. Behind the bubble, the liquid film collides with the liquid entrained by bubble that causes strong mixing. A positive shear stress pulsation appears directly behind the bubble as a pulsations zone, which eventually decays to laminar background flow. In flow with $R e=217$, Fig. 3 (d), the length of the pulsations zone behind the bubble is about $l_{\mathrm{p}} / \mathrm{D} \sim 5$. For moderate $R e=954$, Fig. 3 (e), the length increases up to $l_{\mathrm{p}} / \mathrm{D} \sim 35$. For higher Reynolds number, Fig. 3 (f), the length exceeds $l_{\mathrm{p}} / \mathrm{D}=400$. Previously, in literature a bubble wake was investigated in detail in [2]. The wake length was estimated as $(16 \div 30) D$. Later, according to [9], the length increased up to $50 D$.

In the current work $l_{\mathrm{p}}$ considers as the length of the wall shear stress pulsations zone. This length is presented in Fig. 3 (a) for small bubbles, while in Fig. 3 (b) - for Taylor bubbles. The data for Taylor bubbles are divided conditionally in two series for short bubbles $\left(L_{\mathrm{b}} \sim 0.04 \div 0.2 \mathrm{~m}\right)$ and long bubbles $\left(L_{\mathrm{b}} \sim 0.25 \div 0.65 \mathrm{~m}\right)$. For small bubbles the pulsations zone length $-l_{\mathrm{p}} / D$ increased point-by-point with the liquid Reynolds number. The maximal length is about $70 \div 90 D$ that significantly exceeds even the Taylor bubble 
wake length indicated in the literature. The data for Taylor bubbles length depends on the liquid Reynolds number. Starting from very small values at small Re numbers, the length sharply increased up to $400 D$ for $\mathrm{Re} \geq 1500$. At higther Re the length for long Taylor bubbles is $1.5 \div 2$ times bigger than that for short ones.
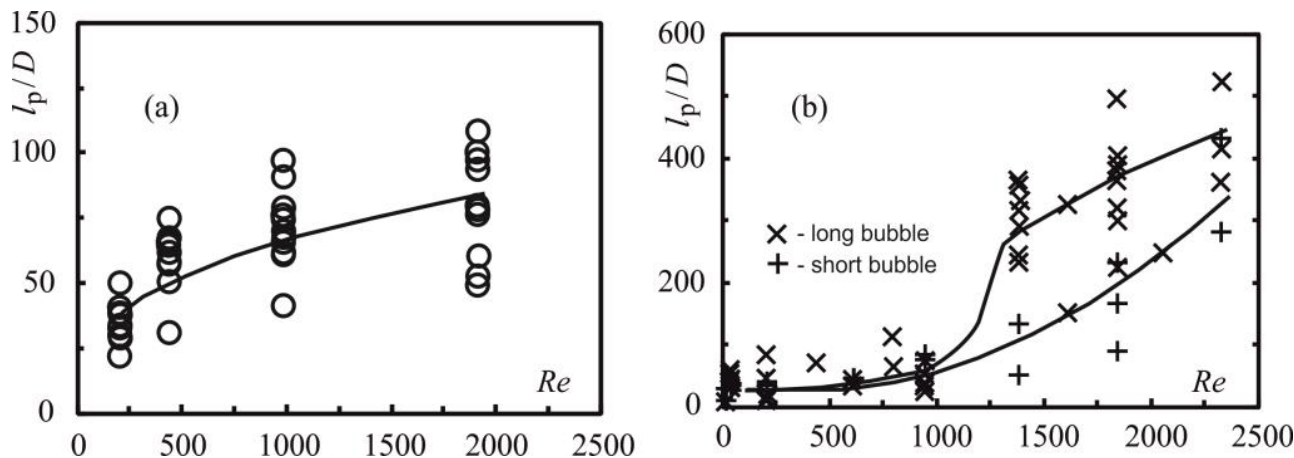

Fig. 3. The pulsations zone length: (a) for almost spherical bubbles with $d_{\mathrm{b}} \sim 3 \div 6 \mathrm{~mm}$; (b) for short Taylor bubbles with lengths of $L_{\mathrm{b}} \sim 0.04 \div 0.2 \mathrm{~m}$ and long bubbles with with lengths of $L_{\mathrm{b}} \sim 0.25 \div$ $0.65 \mathrm{~m}$.

Thus, the length of shear stress pulsations zone significantly depends on the liquid Reynolds number. At high Re numbers for small almost spherical bubbles it achieve values of $(50 \div 90) D$ and becomes drastically long, exceeding more them $400 D$ for long Taylor bubbles that significantly exceeds the length of bubbles wakes, mentioned in literature.

\section{References}

1. D.T. Dumitrescu, Z. Angew. Math. Mech. 23, 139 (1943)

2. R. Moissis, P. Griffith, J. Heat Transfer, 84, 29 (1962)

3. A.O. Morgado, J.M. Miranda, J.D.P. Araújo, J.B.L.M. Campos, Int. J. Multiph. Flow 85, 348 (2016)

4. V.E. Nakoryakov, L.S. Timkin, R.S. Gorelik, Thermophys. Aeromech. 18, 281 (2011)

5. L.S. Timkin, R.S. Gorelik, P.D. Lobanov, J. Eng. Phys. Thermophys. 78, 762 (2005)

6. G. Hetsroni, C.F. Li, A. Mosyak, I. Tiselj, Int. J. Multiphase Flow 27, 1127 (2001)

7. M.N. Descamps, R.V.A. Oliemans, G. Ooms, R.F. Mude, Exp. Fluids 45, 357 (2008)

8. R. Van Hout, A. Gulitski, D. Barnea, L. Shemer, Int. J. Multiphase Flow 28, 579 (2002)

9. J.D.P. Araujo, J.M. Miranda, A.M.F.R. Pinto, J.B.L.M. Campos, Int. J. Multiphase Flow 43, 131 (2012) 